\title{
THE BIOSENTINEL BIOANALYTICAL MICROSYSTEM: CHARACTERIZING DNA RADIATION DAMAGE IN LIVING ORGANISMS BEYOND EARTH ORBIT
}

\author{
A.J. Ricco, ${ }^{l}$ R. Hanel, ${ }^{l}$ S. Bhattacharya, ${ }^{l}$ T. Boone, ${ }^{l}$ M. Tan, ${ }^{l}$ A. Mousavi, ${ }^{l}$ M. Padgen, ${ }^{l}$ D. Gentry, ${ }^{l}$ \\ A. Rademacher, ${ }^{1}$ A. Schooley, ${ }^{1}$ B. Klamm, ${ }^{l}$ J. Benton, ${ }^{l}$ C. Friedericks, ${ }^{l}$ G. Defouw, ${ }^{l}$ M. Parra, ${ }^{l}$ \\ S. Santa Maria, ${ }^{1}$ D. Marina, ${ }^{1}$ B. Lewis, ${ }^{1}$ H. Sanchez, ${ }^{l}$ J. Chartres, ${ }^{l}$ D. McIntosh, ${ }^{1}$ and T. Lusby, ${ }^{1}$ \\ S. Gavalas, ${ }^{2}$ S. Wheeler, ${ }^{2}$ and the Johnson Space Center Radworks Group ${ }^{2}$ \\ ${ }^{1}$ NASA Ames Research Center, Moffett Field, California, USA \\ ${ }^{2}$ NASA Johnson Space Center, Houston, Texas, USA
}

\begin{abstract}
We report details and initial lab test results from an integrated bioanalytical microsystem designed to conduct the first biology experiments beyond low Earth orbit (LEO) since Apollo 17 (1972). The 14-kg, $12 \times 24 \times 37-\mathrm{cm}^{3}$ BioSentinel spacecraft will assay radiation-responsive yeast in its science payload by measuring DNA double-strand breaks (DSBs) repaired via homologous recombination, a mechanism common to all eukaryotes including humans. S. cerevisiae (brewer's yeast) in 288 microwells are provided with nutrient and optically assayed for growth and metabolism via 3-color absorptimetry periodically during the $\sim 12$-monthlong spaceflight mission. BioSentinel, one of several secondary payloads to be deployed by NASA's Space Launch System (SLS) as it carries Exploration Mission 1 into space in 2018, will receive commands and telemeter data to Earth from tens of millions of $\mathrm{km}$.
\end{abstract}

\section{INTRODUCTION}

Small autonomous satellites, called nanosatellites or cubesats, simultaneously reduce cost and increase accessibility for spacescience experiments [1]. Leveraging and integrating advances in nano-, micro-, and miniature technologies in fields from biotechnology to microfluidics to telecommunications, small satellites are being developed worldwide by over 100 universities, numerous small ventures, several large aerospace companies, multiple developing nations, and all major space agencies. Despite their diminutive size, they support complex science [1-4]. By coupling autonomy and telemetry in these space "free flyers", near-real-time experimental data are provided from environments that may be challenging or costly for human missions [2-4].

For life to live and thrive beyond low Earth orbit (LEO) requires understanding and managing multiple unique perturbations. While the International Space Station (ISS) and other orbiting spacecraft provide reduced- (including) micro-gravity environments, the complex radiation environment of interplanetary space comprises many particle types, each with its own energy spectrum and resultant effective biological dose rate. This complex environment is not reproduced by any terrestrial facility: radiationexposure facilities typically provide one or two particle types at one or two energies simultaneously; a dose equivalent to months in outer space is delivered in a matter of minutes to hours. Because of the capacity of biological systems for self-repair and cell-cell communication, the response to acute vs. chronic dosage cannot be assumed to be identical, even for the same total dose. Thus, complete radiation-response characterization necessitates chronic exposure while monitoring live organisms beyond the shielding effects of Earth's magnetosphere for periods of months [5].

BioSentinel's strategy is that of 'the canary in the coal mine': assess health risk for long-duration human missions beyond LEO using a model organism with important similarities to humans, in this case the mechanism by which DNA double-strand breaks are repaired [6]. Of the categories of biological damage created by low-to-moderate levels of chronic radiation exposure, damage to DNA is a major concern: resulting mutations can cause cancer.

In order to better understand how biological effects can be related to physically measured radiation dosage in the space environment [7], and to help validate biological radiation-effect models, BioSentinel will also carry two types of solid-state radiation sensor: a total ionizing dose (TID) dosimeter and a miniaturized linear-energy-transfer (LET) spectrometer (details below).

BioSentinel's biological radiation experiment utilizes multiple independently actuated sets of microfluidically-serviced microwell arrays to monitor DNA DSBs, measuring their impact on viability as well as their rate of successful repair. Three strains of Saccharomyces cerevisiae are utilized to accomplish this: (1) a wildtype, serving as control, which is minimally affected by the dose range anticipated during the BioSentinel mission; (2) a mutant strain, $\operatorname{rad} 51 \Delta$, which is defective in DNA double-strand break repair by homologous recombination and, therefore, more likely than wildtype yeast to be made non-viable by DSBs; (3) an engineered strain with a genetic defect the prevents it from producing an amino acid (e.g., leucine) essential to its growth. This so-called biosentinel strain of yeast, when fed with growth medium lacking leucine, neither reproduces nor exhibits the levels of metabolic activity associated with growth; however, when its DNA DSB repair process is activated, for example by a high-energy radiation damage event, that genetic defect can be simultaneously repaired. Thus, if even one yeast cell in one microwell suffers a DSB from one radiation 'hit' and successfully repairs its genetic defect, this cell acquires the capacity to synthesize the missing amino acid and, through exponential reproduction, generates readily measurable metabolic activity and population growth in that microwell.

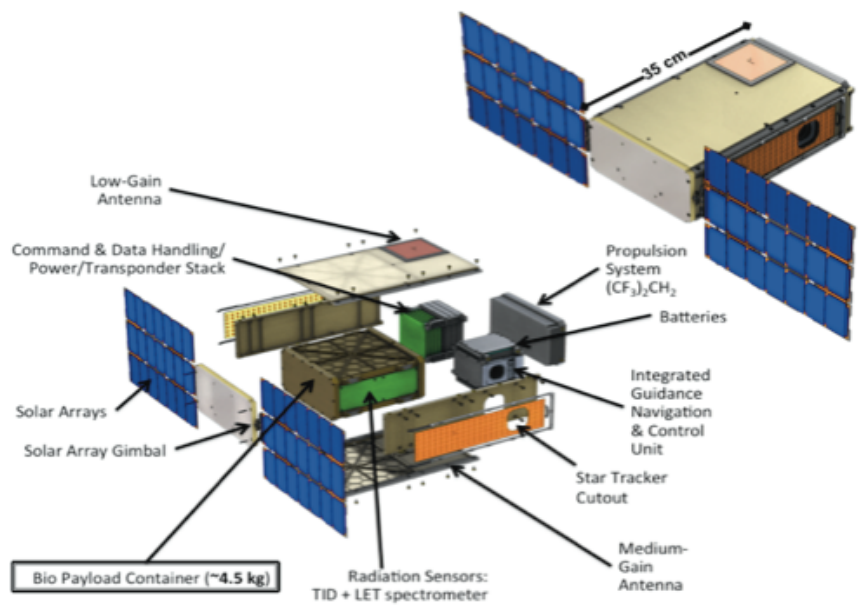

Figure 1: BioSentinel spacecraft views: exploded (left) and assembled (upper right). Solar panels are pointed toward the sun and radio antennas toward Earth by the integral guidance, navigation, and control unit. The bio payload container occupies $\sim 4 \mathrm{~L}$. Hilton Head Island, South Carolina, June 5-9, 2016 


\section{DESIGN AND EXPERIMENTAL DETAILS}

Figure 1 shows a solid model of the BioSentinel spacecraft and its major subsystems and components. Its deployment from the SLS launch vehicle upper stage will place it in a heliocentric, so-called 'disposal orbit' (never to return to Earth) in the range of $0.93-0.97$ astronomical units (AU) from the sun and well beyond Earth's magnetic field and radiation belts. Due to its orbit, BioSentinel's orbital velocity will cause its distance from Earth to increase with time: after 12 months, BioSentinel is expected to be $\sim 10^{8} \mathrm{~km}$ away. It is therefore designed for operation in deep space and features a deployable, gimbaled triple-junction solar panel array that generates up to $35 \mathrm{~W}$; an integrated guidance, navigation, and control (GN\&C) unit, including 3 orthogonally oriented momentum wheels, a star tracker, multiple sun sensors, and an inertial measurement unit; a cold-gas micropropulsion system for detumbling upon initial deployment and for desaturating the momentum wheels; lithium ion batteries and an electric power management system; a radio/transponder and multiple 'patch' antennas for communication with NASA's Deep Space Network of antennas; and overall electronics tolerance through a combination of part selection, localized shielding, and 'watchdog timers' to the radiation dose anticipated in one year or more in deep space. These subsystems, together with the mechanical structures, occupy $\sim$ half of the total spacecraft volume, leaving $\sim 4 \mathrm{~L}$ volume for the experimental-biology-and-radiation-monitoring payload.

Figure 2 shows one of BioSentinel's 18 microfluidic "minicards", fabricated by precision machining, laser/blade cutting, and lamination with pressure-sensitive adhesive (PSA). Each well is $3.5 \mathrm{~mm}$ in diameter $\mathrm{x} 10 \mathrm{~mm}$ deep, for a contained volume of $100 \mu \mathrm{L} /$ well. Integral "track-etched" (Whatman Nucleopore) translucent polycarbonate membranes $(1.0 \mu \mathrm{m}$ pore diameter; a single common layer covers the tops of the minicard microwells and a second common layer covers the bottoms) provide crosstalkfree inlet/outlet filtration, confining the various strains of yeast to their respective microwells while permitting initial microwell filling from the dry state (displacement of air), exchange of medium, and high-precision light-transmission measurements.
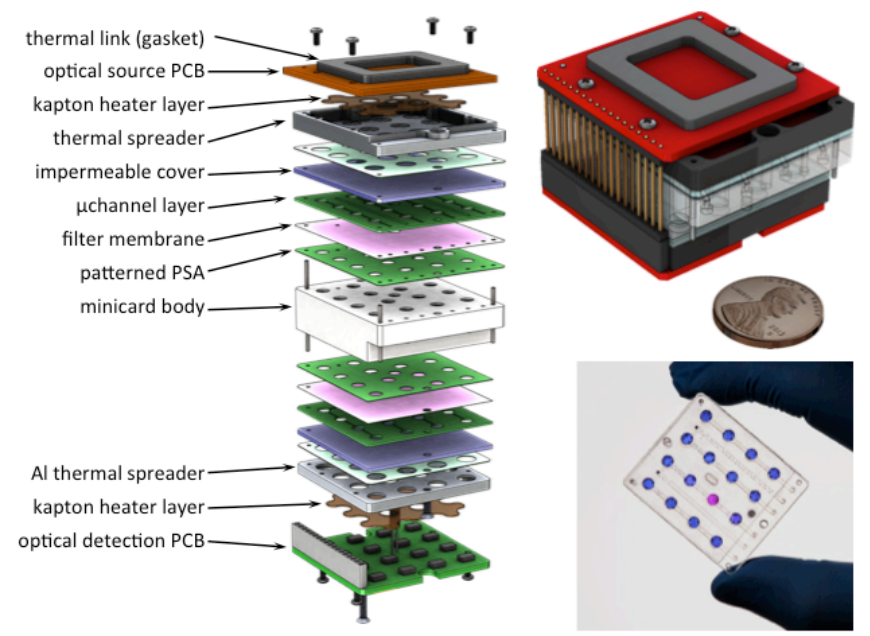

Figure 2: Exploded (left) and assembled (upper right) views of the microfluidic minicard with integral thermal control and optical measurement components. Bottom right: photo of a functional prototype fluidic minicard; 15 wells contain alamar blue and one well contains the same dye that has turned pink due to yeast metabolic activity. (For microwell cross section, see Fig. 3.)
Minicard bodies and microchannel-defining layers are made of polycarbonate, as are the Nucleopore membranes; the acrylic PSA maintains bonding strength at temperatures above $130{ }^{\circ} \mathrm{C}$. Thus, the entire fluidic minicard can be sterilized by autoclaving at $121{ }^{\circ} \mathrm{C}$, a process not typically tolerated by thermoplastic microfluidic systems but which is simple and provides reliable sterility.

After minicard sterilization, yeast cells are dried onto microwell walls in preparation for spaceflight; cards are sealed and integrated with a fluid-supply manifold (Fig. 2) and the entire system is integrated, including reagents and media contained in fluoroethylene polymer bags (American Fluoroseal), an integrated fluid-delivery system, control electronics, and MEMS pressure (Freescale) and humidity (Sensirion) sensors. The integrated biosensor system is sealed inside the hermetic bio payload container.

When properly dried, yeast can survive for months to years. This is critical because the fully integrated BioSentinel spacecraft will be delivered to the launch provider several months prior to its launch into space; also, the mission itself has a nominal one-year duration, with the expectation that one or more minicards will be activated (i.e., their yeast will be grown) near the end of this time.

To help ensure the longevity of the dried yeast while awaiting activation, BioSentinel includes a 'refrigeration' feature. One large face of the spacecraft (Fig. 1) is pointed, whenever feasible, towards dark space; it is coated to enhance thermal emissivity. The integrated fluidic minicards, Fig. 2, are topped by a conformal thermally conductive gasket pressed into contact with the inside face of the container that is thermally coupled to the cold spacecraft face. Thermal modeling suggests a temperature of $\sim 4-8{ }^{\circ} \mathrm{C}$ can be maintained within the unheated minicards - which are monitored and heated only if necessary to avoid freezing - dependent on the details of the orbit and spacecraft operation. Only the minicard(s) with wetted yeast in the active growth phase are thermostatted at the higher growth temperature, $\sim 20{ }^{\circ} \mathrm{C}$.

Figure 3 , a cross-section of a single fluidic well, shows the optical measurement and thermal control components. Three surface-mount LEDs provide illumination $(570,630$, and $850 \mathrm{~nm}$, sequentially); absorbances are calculated from the output of a dedicated intensity-to-frequency light sensor (ams/TAOS) at each well (the optical system has zero moving parts). Minicard cover layers are optical-quality, permeation-resistant poly(cycloolefin) (1-mmthick Zeonor injection-molded plaques).

The lower part of Fig. 3 shows the design of a nine-minicard manifold that provides fluid in/out connections, houses solenoid valves and check valves, and supports an optical calibration cell (visible at the near end of the lower bank of minicards). This cell is used to measure the absorbance of alamar blue to confirm its concentration and provide an optical calibration datum at all three wavelengths for each minicard at its time of filling.

The 16-well fluidic minicards will be activated either singly or in pairs across the duration of the one-year mission in deep space in order to quantify time-dependent accumulated DNA DSBs. Fluid filling and metering are accomplished by a miniature peristaltic pump (Takasago) and 3-way solenoid valves (Lee Co.). $S$. cerevisiae grow after microwells have been filled with a 9:1 mix of synthetic complete (SC) growth medium (minus one deliberately omitted amino acid, as described above) and alamar blue indicator dye. This dye is chemically reduced from dark blue to (fluorescent) pink, then to colorless, due to cellular metabolic activity that produces reducing agents such as NADH and FADH $[2,4]$.

In addition to its bioanalytical microsystem, BioSentinel includes two physical radiation sensors to measure total ionizing dose and linear energy transfer. Given the shielding provided by the spacecraft structure, the estimated one-year accumulated ionizing radiation dose inside the payload is estimated at $5 \mathrm{~Gy}$. The 
TID dosimeter (Teledyne uDOS001; volume $9.2 \mathrm{~cm}^{3}$; mass $20 \mathrm{~g}$ ) is a silicon-based solid-state charge-integrator-based device that has been utilized for multiple space missions. It features a resolution of $140 \mathrm{nGy}$ (the dose received, on average, in $\sim 0.9 \mathrm{sec}$ during the BioSentinel mission) and full-scale of $400 \mathrm{~Gy}$.

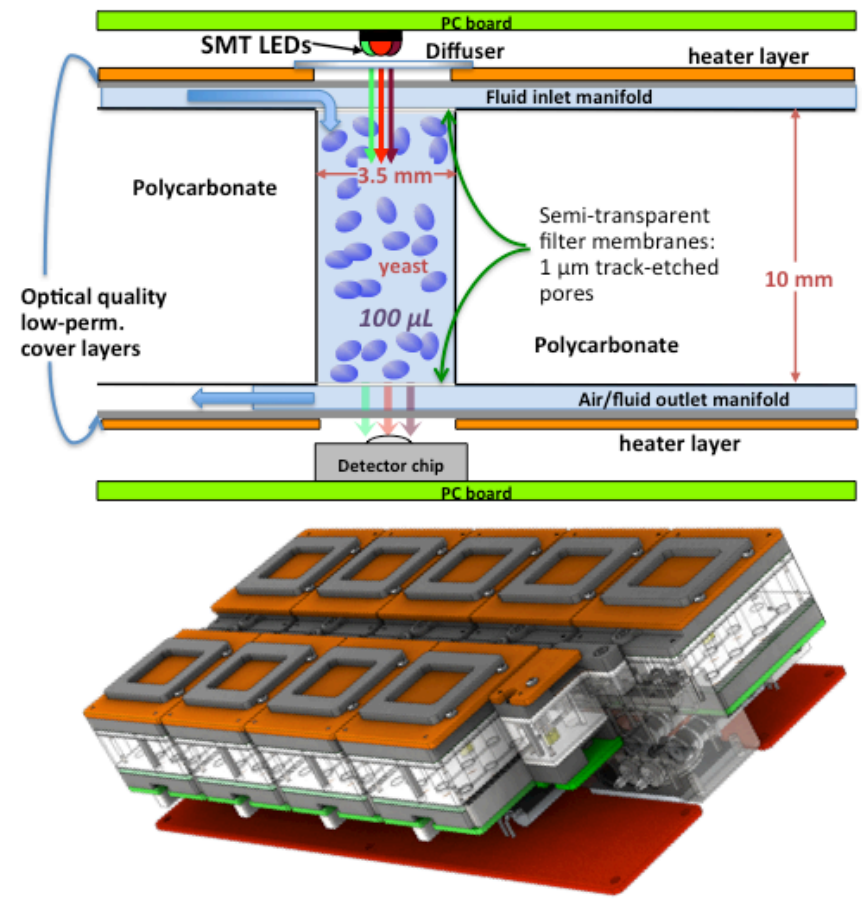

Figure 3: Top: Cross section of one fluidic microwell with integral filter membranes and connecting microchannels. Yeast dried onto walls grow and bud when medium is introduced, displacing air through porous membranes. Three LEDs per well, with an intervening thin-film polymer diffuser, provide green, red, and nearinfrared (IR) illumination to monitor metabolic activity and track yeast growth via an intensity-to-frequency detector at the bottom of each well. Patterned metal-on-kapton heaters with aluminum thermal spreader plates maintain uniform, stable growth temperature $\left(\sim 20 \pm 1{ }^{\circ} \mathrm{C}\right)$ via closed-loop control; a Pt resistancetemperature device is embedded in each minicard. Bottom: Nine fluidic minicards will be integrated and manifolded together for spaceflight; two such sets (288 microwells in total) will be included in the hermetically sealed $4 L$ bio payload container (Fig. 1).

The LET spectrometer, developed and provided by Johnson Space Center's Radworks group, is based closely on a predecessor that has flown on the ISS as well as the first test flight of NASA's Orion spacecraft in 2014. The charge-coupled Si device records the passage of each high-energy particle as a track or 'blob' of pixels with accumulated charge (Figure 4). The associated controland-measurement electronics and software convert the track/blob data into the energy deposited by each high-energy particle per unit length of Si traversed, thus defining its potential to cause (biological) damage. Closed-loop feedback adjusts the integration time to keep frame pixel occupancy $\leq 3 \%$, making deconvolution of the tracks (more) straightforward. The sensor measures radiation over the 0.2 to $300 \mathrm{keV} / \mu \mathrm{m}$ LET range, which is most relevant for biological damage, using an active chip volume of $59 \mathrm{~mm}^{3}$. On-board software computes the LET value of each radiation event (particle hit) and increments the count in an appropriate bin; each of the 256 counting bins is defined by an LET width of $\sim 2.9 \%$ of its center value. The binned event counts are reported hourly by the LET spectrometer to the spacecraft's main processor for storage and eventual telemetry to Earth, providing an hourly 'space weather report' of the types and numbers of ionizing particles encountered by BioSentinel.

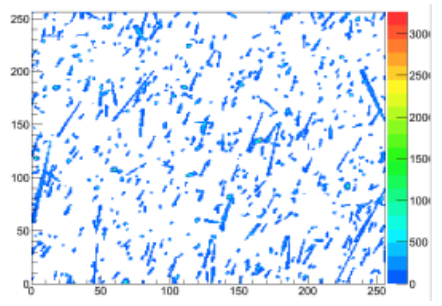

Figure 4: Typical radiationevent data frame recorded by the CCD-based LET sensor; each locus of points is associated with the passage of a single particle or ray.

The inclusion of TID and LET sensors enables the BioSentinel biological experiment to respond to changes in space weather. In particular, during a solar particle event (SPE), radiation flux can spike to a thousand times nominal levels or more; it is therefore a goal of the BioSentinel mission to measure the effects of such an event, should it occur during the year-long mission. Yeast (and other cells) are generally significantly more damaged by radiation when hydrated than when dry, at least in part because $\mathrm{OH} \bullet$ species, created from water by radiation, cause oxidative damage. With 18 fluidic minicards to be tested periodically across 12 months, and a growth duration measured in hours, there may or may not be a wet, actively growing minicard ready to respond to the enhanced flux of an SPE. Therefore, onboard algorithms will monitor both TID and LET outputs and, should the flux rise sufficiently above background, a "special" minicard will be activated so that its cells are wet and ready to grow as the main part of the SPE arrivestypically a fraction of one to a few hours after radiation levels initially begin to surge.

\section{PRELIMINARY LABORATORY RESULTS}

Figure 5 shows biological growth and metabolism results from laboratory testing of a flight prototype of the integrated optical/fluidic/bioanalytical system. The results are for wildtype yeast grown in SC medium at room temperature in the presence of " $1 \mathrm{x}$ " alamar blue viability dye. As growth progresses, green absorbance increases as the bright pink color of reduced alamar blue appears, then decreases as pink fades to colorless. Red absorbance decreases as the blue dye turns pink, then increases due to cell population growth. Near-IR absorbance, unaffected by all forms of the dye, tracks cell population due to the linear relationship between turbidity and cell number. 16 such curves are produced simultaneously from one growing fluidic minicard.

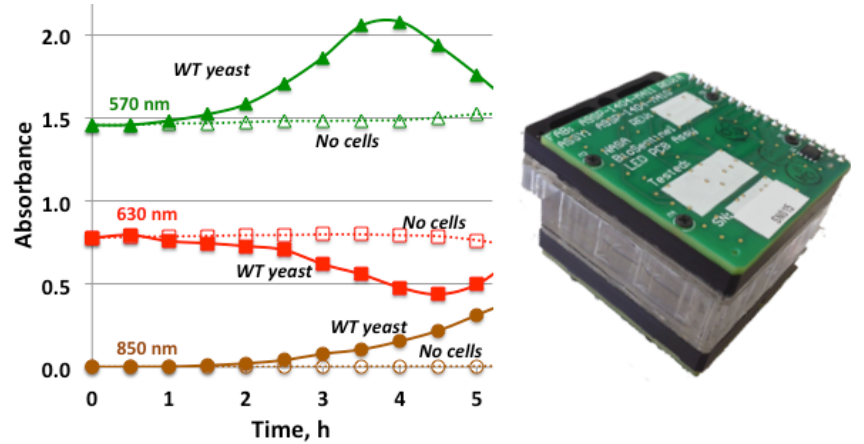

Figure 5: Growth curves (left) recorded using spaceflight optical/fluidic/thermal prototype shown in the photograph (right) for wildtype (WT) S. cerevisiae with added alamar blue (filled symbols) and cell-free control microwells (open symbols); LED wavelengths are as indicated. 


\section{CONCLUSIONS}

Directly studying biology's response to the interplanetary space radiation environment has never been attempted apart from a few costly, human-operated beyond-LEO missions. Robust, autonomous bioanalytical microsystems compatible with small satellites are poised to change this paradigm. BioSentinel is supported by NASA's Advanced Exploration Systems division.

\section{REFERENCES}

[1] K. Woellert, P. Ehrenfreund, A.J. Ricco, and H. Hertzfeld, "Cubesats: Cost-Effective Science and Technology Platforms for Developing Nations," Adv. Space Res., 47, 663 (2011).

[2] W. L. Nicholson, A.J. Ricco, E. Agasid, C. Beasley, M. DiazAguado, P. Ehrenfreund, C. Friedericks, S. Ghassemieh, M. Henschke, J. W. Hines, C. Kitts, E. Luzzi, D. Ly, N. Mai, R. Mancinelli, M. McIntyre, G. Minelli, M. Neumann, M. Parra, M. Piccini, R. Rasay, R. Ricks, O. Santos, A. Schooley, D. Squires, L. Timucin, B. Yost, A. Young, "The O/OREOS Mission: First Science Data from the SESLO Payload," Astrobiology, 11, 951 (2011).

[3] A. Mattioda, A. Cook, P. Ehrenfreund, R. Quinn, A.J. Ricco, D. Squires, N. Bramall, K. Bryson, J. Chittenden, G. Minelli, E. Agasid, L. Allamandola, C. Beasley, R. Burton, G. Defouw, M. Diaz-Aguado, M. Fonda, C. Friedericks, C. Kitts, D. Landis, M. McIntyre, M. Neumann, M. Rasay, R. Ricks, F. Salama, O. Santos, A. Schooley, B. Yost, and A. Young "The O/OREOS Mission: First Science Data from the Space Environment Viability of Organics (SEVO) Payload," Astrobiology, 12, 841 (2012).

[4] A.J. Ricco, M. Parra, D. Niesel, M. Piccini, D. Ly, M. McGinnis, A. Kudlicki, J.W. Hines, L. Timucin, C. Beasley, R. Ricks, M. McIntyre, C. Friedericks, M. Henschke, R. Leung, M. Diaz-Aguado, C. Kitts, I. Mas, M. Rasay, E. Agasid, E. Luzzi, K. Ronzano, D. Squires, B. Yost, "PharmaSat: Drug Dose Response in Microgravity from A Free-Flying Integrated Biofluidic/Optical Culture-and-Analysis Satellite," Proc. SPIE 7929, Microfluidics, BioMEMS, and Medical Microsystems IX, 79290T (9 pp.), SPIE: Bellingham, WA (2011).

[5] M. Hada and B.M. Sutherland, "Spectrum of complex DNA damages depends on the incident radiation," Radiation Research, 165, 223 (2006).

[6] T.C. Akpa, K.J. Weber, E. Schneider, J. Kiefer, M. Frankernberg-Schwager, R. Harbich, and D. Frankenberg, "Heavy ioninduced DNA double-strand breaks in yeast," International Journal of Radiation Biology, 62, 279 (1992).

[7] H.D. Pross, A. Casares, and J. Kiefer, "Induction and repair of DNA double-strand breaks under irradiation and microgravity," Radiation Research, 153, 521 (2000).

\section{CONTACT}

*A.J. Ricco, tel: +1-650-604-4276; ajricco@stanford.edu 